\title{
Inequivalence of pure state ensembles for open quantum systems: the preferred ensembles are those that are physically realizable
}

\author{
H.M. Wiseman ${ }^{1,2}$, John A. Vaccaro ${ }^{2}$ \\ ${ }^{1}$ Centre for Quantum Dynamics, School of Science, Griffith University, Brisbane, Queensland 4111 Australia. \\ ${ }^{2}$ Division of Physics and Astronomy, University of Hertfordshire, Hatfield AL10 9AB, UK.
}

\begin{abstract}
An open quantum system in steady state $\hat{\rho}_{\mathrm{ss}}$ can be represented by a weighted ensemble of pure states $\hat{\rho}_{\mathrm{ss}}=\sum_{k} \wp_{k}\left|\psi_{k}\right\rangle\left\langle\psi_{k}\right|$ in infinitely many ways. A physically realizable (PR) ensemble is one for which some continuous measurement of the environment will collapse the system into a pure state $|\psi(t)\rangle$, stochastically evolving such that the proportion of time for which $|\psi(t)\rangle=\left|\psi_{k}\right\rangle$ equals $\wp_{k}$. Some, but not all, ensembles are PR. This constitutes the preferred ensemble fact. We present the necessary and sufficient conditions for a given ensemble to be PR, and illustrate the method by showing that the coherent state ensemble is not $\mathrm{PR}$ for an atom laser.
\end{abstract}

03.65.Yz, 03.65.Ta, 42.50.Lc, 03.75.Fi

Introduction. It is well known that there are infinitely many ways to write a given mixed state as a mixture of (in general non-orthogonal) pure states. That is, there are infinitely many ensembles, $\left\{\left(\hat{\Pi}_{k}, \wp_{k}\right)\right\}_{k}$, consisting of ordered pairs of pure states (rank-one projectors $\left.\hat{\Pi}_{k}=\left|\psi_{k}\right\rangle\left\langle\psi_{k}\right|\right)$ and weights $\wp_{k}$, that represent a given state matrix (density operator) $\hat{\rho}$ according to

$$
\hat{\rho}=\sum_{k} \wp_{k} \hat{\Pi}_{k} .
$$

Any such ensemble representation suggests an ignorance interpretation of the state matrix $\hat{\rho}$. That is, one would claim that the system "really" is in one of the pure states $\hat{\Pi}_{k}$, but that one happens to be ignorant of which state $\hat{\Pi}_{k}$ (i.e. which $k$ ) pertains. The weight $\wp_{k}$ is interpreted as the probability that the system has state $\hat{\Pi}_{k}$.

This interpretation can only be maintained for socalled proper mixtures, i.e. those for which the system is not entangled with its environment [1]. However, an improper mixture may be turned into a proper mixture simply by appropriately measuring the environment, and ignoring the result. The partition ensemble fallacy [2] or preferred ensemble fallacy is that one may use a particular ensemble to draw inferences about an experiment, even if those inferences depend upon the choice of ensemble. Here we do not deny that this is indeed a fallacy.

Mixed states arise naturally in the description of open quantum systems, in fields as diverse as chemical physics, quantum optics, micromechanics and nano-electronics. It is often possible to approximate the evolution of such systems by a Markovian master equation $\dot{\hat{\rho}}=\mathcal{L} \hat{\rho}$, where $\mathcal{L}$ is the Lindbladian [3]. Under these conditions, an experimenter may perform continual measurements on the environment with which the system interacts without affecting the master equation evolution [3,4]. Hence the system state can be considered a proper mixture.

Let us restrict the discussion to a stationary mixed state $\hat{\rho}_{\mathrm{ss}}$, assumed unique:

$$
\dot{\hat{\rho}}_{\mathrm{ss}}=\mathcal{L} \hat{\rho}_{\mathrm{ss}}=0 .
$$

Also, let us consider only stationary ensembles for $\hat{\rho}_{\text {ss }}$. Clearly, once the system has reached steady-state then such a stationary ensemble will represent the system for all times $t$. Now, if the ignorance interpretation were to hold for such an ensemble then it should be possible, in principle, for the experimenter to know which state $\hat{\Pi}_{k}$ pertains to the system at any particular time $t$. The pertinent index $k$ would change stochastically such that the proportion of time the system has state $\hat{\Pi}_{k}$ is $\wp_{k}$.

In this letter we show that for some ensembles this ignorance interpretation fails. That is, there is no way that an experimenter can know at all (long) times that the system has some ensemble state $\hat{\Pi}_{k}$. We say that such ensembles are not physically realizable (PR). However, there are other stationary ensembles that are PR.

The existence, for a given system, of two non-empty classes of stationary ensembles, those that are PR and those that are not, constitutes a preferred ensemble fact (PE-fact). Note that this fact identifies a preferred class of ensembles (the PR ones), not a unique preferred ensemble. Also note that this fact does not contradict the argument against preferred ensembles in Ref. [2], because it is a fact about ensembles representing a stationary state for all times. As we will see, the PE-fact has surprising implications for some open quantum systems.

This letter is organized as follows. First we discuss the Hughston-Josza-Wootters theorem [5] and why it does not contradict the PE-fact. Then we give the necessary and sufficient conditions for an ensemble to be PR for a given system. For linear systems with uniform Gaussian ensembles we show these conditions reduce to a simple inequality. Next we establish the PE-fact for a particular system of interest: a model atom laser. Finally we discuss the implications of our results.

The HJW Theorem. We wish to consider a system with state matrix $\hat{\rho}$ which is mixed solely due to its entanglement with a second system, the environment. That is, there is always a pure state $|\Psi\rangle$ in a larger Hilbert space of system plus environment such that 


$$
\hat{\rho}=\operatorname{Tr}_{\mathrm{env}}[|\Psi\rangle\langle\Psi|] .
$$

Certainly it is always possible formally to find such a pure state [5]. Also, if the environment is initially not pure then it can be then measured in its diagonal basis so as to make it conditionally pure without affecting its subsequent interaction with the system. Therefore we can assume the existence of $|\Psi\rangle$ without loss of generality.

The Hughston-Jozsa-Wootters (HJW) theorem [5] says the following. For any ensemble $\left\{\left(\hat{\Pi}_{k}, \wp_{k}\right)\right\}_{k}$ that represents $\hat{\rho}$, it is possible to measure the environment such that the system state is collapsed into one of the pure states $\hat{\Pi}_{k}$ with probability $\wp_{k}$. That is, there exists a probability-operator measure $\left\{\hat{F}_{k}\right\}_{k}$ acting on the environment Hilbert space such that

$$
\wp_{k} \hat{\Pi}_{k}=\operatorname{Tr}_{\text {env }}\left[\hat{1}_{\text {sys }} \otimes \hat{F}_{k}^{1 / 2}|\Psi\rangle\langle\Psi| \hat{F}_{k}^{1 / 2} \otimes \hat{1}_{\text {sys }}\right] .
$$

The HJW theorem gives rigorous meaning to the ignorance interpretation of any particular ensemble. It says that there will be a way to perform a measurement on the environment, without on average disturbing the system state, to obtain exactly the information as to which state the system is "really" in. Of course the fact that one can do this for any ensemble means that no ensemble can be fundamentally preferred over any other one, as a representation of $\hat{\rho}$ at some particular time $t$.

The HJW theorem does not contradict the preferred ensemble fact introduced above. This is because the PEfact refers to the much stronger notion of representing the state of the system obeying a master equation, at steady state, by a stationary ensemble that applies at all times. This extra condition means that the HJW theorem does not apply, and not all ensembles are PR through measuring the environment of the system. We will now give the conditions for an ensemble to be PR.

Establishing the Preferred Ensemble Fact. Consider a system obeying the master equation $\dot{\hat{\rho}}=\mathcal{L} \hat{\rho}$ in the longtime limit so that it is described by the (assumed unique) stationary state $\hat{\rho}_{\mathrm{ss}}$. Now say we wish to give an ignorance interpretation at all long times to a particular stationary ensemble $\left\{\left(\hat{\Pi}_{k}, \wp_{k}\right)\right\}_{k}$ satisfying

$$
\hat{\rho}_{\mathrm{ss}}=\sum_{k} \wp_{k} \hat{\Pi}_{k} .
$$

At a particular time $t$ this is always possible by the HJW theorem. That is, we can measure the environment to find out the pertinent state $\hat{\Pi}_{k}$, and, on average, the system remains in state $\hat{\rho}_{\mathrm{ss}}$. This may involve measuring parts of the environment that interacted with the system an arbitrarily long time ago, but there is nothing physically impossible in doing this.

Now consider the future evolution of the system state following this measurement. At time $t+\tau$, it will have evolved to $\hat{\rho}_{k}(t+\tau)=\exp (\mathcal{L} \tau) \hat{\Pi}_{k}$. This is a mixed state because the system has now become re-entangled with its environment. The system state can be repurified by making another measurement on its environment. However, if the same ensemble is to remain as our representation of the system state then the pure system states obtained as a result of this measurement at time $t+\tau$ must be contained in $\left\{\hat{\Pi}_{k}\right\}_{k}$. By the HJW theorem, this will possible if and only if $\hat{\rho}_{k}(t+\tau)$ can be represented as a mixture of these states. That is, for all $k$ there must exist a probability measure $\left\{w_{j k}(\tau)\right\}_{j}$ such that

$$
\exp (\mathcal{L} \tau) \hat{\Pi}_{k}=\sum_{j} w_{j k}(\tau) \hat{\Pi}_{j} .
$$

If $w_{j k}(\tau)$ exists then it is the probability that the measurement at time $t+\tau$ yields the state $\hat{\Pi}_{j}$.

Equation (6) is necessary but not sufficient for the ensemble $\left\{\left(\hat{\Pi}_{j}, \wp_{j}\right)\right\}_{j}$ to be PR. We also require that the weights be stationary. That is, that for all $j$ and all $\tau$,

$$
\wp_{j}=\sum_{k} \wp_{k} w_{j k}(\tau) .
$$

Multiplying both sides of Eq. (6) by $\wp_{k}$, and summing over $k$, then using Eq. (1) and Eq. (5) gives $e^{\mathcal{L} \tau} \hat{\rho}_{\mathrm{sS}}=\hat{\rho}_{\mathrm{ss}}$, as required from the definition of $\hat{\rho}_{\mathrm{ss}}$.

If the two conditions (6) and (7) are satisfied for some time $\tau$ then they will be satisfied for any multiple of $\tau$. Therefore it is sufficient to establish that they are satisfied for $\tau=d t$. (Here $d t$ is infinitesimal with respect to all relevant system time scales but strictly must be long compared to the environment correlation time; the master equation is not valid on any shorter time scale.) For a bounded Lindbladian superoperator $\mathcal{L}$ and distinct states $\left\{\hat{\Pi}_{k}\right\}_{k}$, if $w_{j k}(d t)$ exists then it is given by

$$
w_{j k}(d t)=\delta_{k j}-\delta_{k j} \sum_{l} \gamma_{k l} d t+\gamma_{k j} d t
$$

with $\gamma_{k j}$ finite. The coefficient $\gamma_{k j}$ may be interpreted as the rate for the system to jump from state $\hat{\Pi}_{k}$ to state $\hat{\Pi}_{j}$. That is, the quantum master equation is replaced by a classical master equation [6] for the occupation probabilities $\left\{p_{k}\right\}_{k}$ for the states $\left\{\hat{\Pi}_{k}\right\}_{k}$ where $p_{j}(t+d t)=\sum_{k} p_{k}(t) w_{j k}(d t)$, and so from Eq. (8)

$$
\dot{p}_{k}=-p_{k} \sum_{j} \gamma_{k j}+\sum_{j} \gamma_{k j} p_{j} .
$$

The condition (7) is then equivalent to the condition that $p_{k}=\wp_{k}$ be the stationary solution of Eq. (9).

Any stationary scheme of continual rank-one measurements of the environment will produce stochastic dynamics of this sort in the steady state [7]. The ignorance interpretation of the ensemble thus produced is then rigorously justified. The PE-fact follows if, for the given system, there exists at least one ensemble satisfying Eq. (5) for which there does not exist a probability measure $\left\{w_{j k}(\tau)\right\}_{j}$ satisfying conditions (6) and (7). 
The replacement of a quantum master equation by a classical master equation where the classical index $k$ is associated with a quantum state $\hat{\Pi}_{k}$ is often used to provide an intuitive picture of irreversible quantum dynamics. A canonical example is Einstein's description [8] of an atom driven by a thermal field in terms of jumps from ground to excited states (absorption) and from excited states to ground (emission). Of course Einstein did not know the more general description in terms of the quantum master equation, but it is easy to verify that the the ensemble consisting of atomic energy eigenstates is a PR ensemble. Specifically, to realize this ensemble one must continually measure the environment in the photon number basis.

Linear Dynamics and Uniform Gaussian Ensembles. The description given above applies most naturally to ensembles with a discrete set of states $\left\{\hat{\Pi}_{k}\right\}_{k}$. In many cases we wish to consider a continuum of states. In these cases it is convenient to take a limit in which the jump process described by rates $\gamma_{k j}$ is replaced by a diffusion process. We restrict ourselves to systems with linear dynamics, and uniform Gaussian ensembles. These terms (defined below) only apply to quantum systems whose state $\hat{\rho}$ can be represented by a Wigner function [3] on $2 n$-dimensional Euclidean phase space:

$$
W(\vec{z})=\operatorname{Tr}\left[\hat{\rho} S \prod_{m=1}^{2 n} \int \frac{d \xi_{m}}{2 \pi} \exp \left[i \xi_{m}\left(z_{m}-\hat{z}_{m}\right)\right]\right]
$$

Here $S$ denotes ordering symmetric in the operators

$$
\left(\hat{z}_{1}, \cdots \hat{z}_{2 n}\right)=\left(\hat{x}_{1}, \hat{p}_{1}, \hat{x}_{2}, \hat{p}_{2}, \cdots, \hat{x}_{n}, \hat{p}_{n}\right)
$$

where the co-ordinates $\left\{\hat{x}_{n}\right\}_{n}$ and momenta $\left\{\hat{p}_{n}\right\}_{n}$ each form a mutually commuting set of operators with the reals as eigenvalues, but (with $\hbar=2)\left[\hat{x}_{n}, \hat{p}_{n^{\prime}}\right]=2 i \delta_{n, n^{\prime}}$.

Such a system has linear dynamics if and only if (for a suitable choice of co-ordinates and momenta) its Wigner function obeys an Ornstein-Uhlenbeck equation [6]

$$
\dot{W}(\vec{z})=\left(\vec{\nabla}^{T} \mathbf{K} \vec{z}+\frac{1}{2} \vec{\nabla}^{T} \mathbf{D} \vec{\nabla}\right) W(\vec{z})
$$

Here $\nabla_{m}=\partial / \partial z_{m}, \mathbf{K}$ is a constant matrix, and $\mathbf{D}$ is a constant matrix that is symmetric and positive semidefinite. For simplicity we will assume that the eigenvalues of $\mathbf{K}$ have positive real parts, so that the system has a stationary state

$$
W_{\mathrm{ss}}(\vec{z})=G\left(\vec{z} ; \overrightarrow{0}, \mathbf{V}_{\mathrm{ss}}\right)
$$

where $\mathbf{V}_{\mathrm{ss}}$ is defined by 6

$$
\mathbf{K} \mathbf{V}_{\mathrm{ss}}+\mathbf{V}_{\mathrm{ss}} \mathbf{K}^{T}=\mathbf{D}
$$

Here we are using the notation that $G(\vec{z} ; \vec{\mu}, \mathbf{V})$ is a multivariate Gaussian in $\vec{z}$ parameterized by the mean vector $\vec{\mu}$ and the covariance matrix $\mathbf{V}[6]$.
A uniform Gaussian ensemble of pure states $\left\{\left(\hat{\Pi}_{k}, \wp_{k}\right)\right\}_{k}$ comprises states $\hat{\Pi}_{k}$ that have Wigner functions $W_{k}(\vec{z})$ that are Gaussians

$$
W_{k}(\vec{z}) \equiv W\left(\vec{z} ; \vec{\mu}_{k}\right)=G\left(\vec{z} ; \vec{\mu}_{k}, \mathbf{V}\right)
$$

with mean determined by $k$ but variance independent of $k$. That is, the states all have the same "shape", but have different displacements in $2 n$-dimensional phase space. Since the ensemble represents $\hat{\rho}_{\mathrm{ss}}$ we have (converting from the discrete $k$ to the continuum variable $\vec{\mu}$ )

$$
W_{\mathrm{ss}}(\vec{z})=\int d \mu_{1} \cdots \int d \mu_{2 n} \wp(\vec{\mu}) W(\vec{z} ; \vec{\mu}) .
$$

Since $W_{\mathrm{ss}}(\vec{z})$ and $W(\vec{z} ; \vec{\mu})$ are Gaussians, $\wp(\vec{\mu})$ is also:

$$
\wp(\vec{\mu})=G\left(\vec{\mu} ; \overrightarrow{0}, \mathbf{V}_{\mathrm{ss}}-\mathbf{V}\right) .
$$

Now consider the conditions for the uniform Gaussian ensemble to be PR, starting with Eq. (6). From Eq. (12), if the system begins with Wigner function $W(\vec{z} ; \vec{\mu})$ then after an infinitesimal time $d t$ its Wigner function will be

$$
G\left(\vec{z} ; \vec{\mu}-\mathbf{K} \vec{\mu} d t, \mathbf{V}+\mathbf{D} d t-\mathbf{K V} d t-\mathbf{V K}^{T} d t\right)
$$

This can be written as a mixture of the ensemble states

$$
\int d \vec{\mu}^{\prime} w\left(\vec{\mu}^{\prime} ; \vec{\mu} ; d t\right) W(\vec{z} ; \vec{\mu})
$$

if and only if the transition probability density is

$$
w\left(\vec{\mu}^{\prime} ; \vec{\mu} ; d t\right)=G\left(\vec{\mu}^{\prime} ; \vec{\mu}-\mathbf{K} \vec{\mu} d t, \mathbf{D} d t-\mathbf{K V} d t-\mathbf{V K}^{T} d t\right)
$$

Since the ensemble is specified completely by $\mathbf{V}$, Eq. (6) turns into the condition on $\mathbf{V}$ that

$$
\mathbf{B}_{\mathbf{V}} \equiv \mathbf{D}-\mathbf{K V}-\mathbf{V} \mathbf{K}^{T} \geq 0
$$

An equivalent condition has been considered by Diósi and Kiefer [9] in a related context, but they did not make the connection with physical realizability and measurements.

Equation (20) implies that the quantum master equation in the Wigner representation (12) is equivalent to a stochastic process for the mean displacements $\vec{\mu}$ of the uniform Gaussian states with Wigner functions $W(\vec{z} ; \vec{\mu})$. The probability distribution for $\vec{\mu}$ is governed by

$$
\dot{p}(\vec{\mu})=\left(\vec{\nabla}^{T} \mathbf{K} \vec{\mu}+\frac{1}{2} \vec{\nabla}^{T} \mathbf{B}_{\mathbf{V}} \vec{\nabla}\right) p(\vec{\mu}),
$$

where here $\nabla_{m}=\partial / \partial \mu_{m}$. This is analogous to Eq. (9).

To establish that condition (17) is also satisfied we thus have to show that the stationary solution of Eq. (22) is $p(\vec{\mu})=\wp(\vec{\mu})$. From Eq. 22 we get [6]

$$
p_{\mathrm{sS}}(\vec{\mu})=G(\vec{\mu} ; \overrightarrow{0}, \mathbf{U}),
$$

where $\mathbf{U}$ is defined by $\mathbf{K U}+\mathbf{U K}^{T}=\mathbf{B}_{\mathbf{V}}$. Now using Eqs. (14) and (21) we obtain 


$$
\mathbf{U}=\mathbf{V}_{\mathrm{ss}}-\mathbf{V} .
$$

From Eq. (17) it follows that $p_{\mathrm{ss}}(\vec{\mu})=\wp(\vec{\mu})$ as desired.

Example: The Atom Laser. We now apply the above formalism to the problem of an atom laser. We consider only a CW atom laser (which has not yet been realized), which would consist of a continuously damped and replenished Bose-Einstein condensate. The damping at rate $\kappa$ would produce a continuous beam of coherent atoms [10]. The simplest quantum model for such a device uses a single mode description of the condensate, with annihilation operator $\hat{a}=(\hat{x}+i \hat{p}) / 2$. Assuming a Poissonian pump with rate $\kappa \mu$, the stationary state of the laser is 10,11.

$$
\hat{\rho}_{\mathrm{SS}}=\int \frac{d \phi}{2 \pi}\left|\sqrt{\mu} e^{i \phi}\right\rangle\left\langle\sqrt{\mu} e^{i \phi}\left|=\sum_{n=0}^{\infty} e^{-\mu} \frac{\mu^{n}}{n !}\right| n\right\rangle\langle n|,
$$

Here $\left|\sqrt{\mu} e^{i \phi}\right\rangle$ is a coherent state (eigenstate of $\hat{a}$ ) 11.

Equation (25) shows two of the infinitude of different ensemble representations of $\hat{\rho}_{\mathrm{ss}}$. The number state ensemble $\{|n\rangle\}_{n}$ is clearly PR by making continual measurements of the environment in the atom number basis. On the other hand, to determine the status of the coherent state ensemble $\left\{\left|\sqrt{\mu} e^{i \phi}\right\rangle\right\}_{\phi}$, it is necessary to examine the dynamics of the atom laser.

The simplest reasonable model for an atom laser comes from taking the standard ideal optical laser model [10,12 and adding the interaction between condensate atoms [13], governed by the Hamiltonian $(\hbar \kappa / 4 \mu) \chi \hat{a}^{\dagger 2} \hat{a}^{2}$, with

$$
\chi=\frac{8 \pi \mu \hbar a_{s}}{\kappa m} \int d^{3} \mathbf{r}|\psi(\mathbf{r})|^{4} .
$$

Here $\psi(\mathbf{r})$ is the wavefunction for the condensate mode, and $a_{s}$ is the $s$-wave scattering length. This nonlinear Hamiltonian is difficult to treat exactly, so we linearize the Lindbladian evolution about a mean field $\langle\hat{a}\rangle=\sqrt{\mu}$ (as appropriate for considering the physical realizability of the coherent state). We have shown elsewhere [13] that this results in linear quantum dynamics for the Wigner function $W(x, p)$ as in Eq. (12) with

$$
\mathbf{K}=\kappa\left(\begin{array}{cc}
1 & 0 \\
\chi & 0
\end{array}\right) ; \quad \mathbf{D}=\kappa\left(\begin{array}{cc}
2 & 0 \\
0 & 2+\nu
\end{array}\right)
$$

Here $x$ and $p$ represent amplitude and phase fluctuations respectively, and $\nu \geq 0$ is the excess phase diffusion.

To check if the coherent state ensemble is PR we simply need to check the condition (21). We find

$$
\mathbf{V}=\left(\begin{array}{ll}
1 & 0 \\
0 & 1
\end{array}\right) \Longrightarrow \mathbf{B}_{\mathbf{V}}=\kappa\left(\begin{array}{cc}
0 & -\chi \\
-\chi & 2+\nu
\end{array}\right)
$$

The matrix $\mathbf{B}_{\mathbf{V}}$ is positive semidefinite only for $\chi=0$. That is, the coherent state ensemble is not PR for any finite atomic interaction energy.
Discussion. In this letter we have introduced the necessary and sufficient conditions for an ensemble of pure states to be physically realizable (PR) as a stationary description of a Markovian system in the long-time limit. Here physically realizable means that, by measuring the environment of the system, an experimenter could collapse the system state into a stochastically evolving pure state, such that the proportion of time it is in a particular pure state is equal to the weight of that pure state in the ensemble. For uniform Gaussian ensembles for systems with linear dynamics we derive a simple inequality to distinguish PR ensembles from non-PR ensembles. The existence in general of these non-empty classes of ensembles constitutes the preferred ensemble fact.

To illustrate this fact, we have shown that in a simple model for an atom laser, the number state ensemble is preferred over the coherent state ensemble because only the former is PR. This is due to the presence of atomic interactions, described by a Hamiltonian proportional to the $a^{\dagger 2} a^{2}$. We proved this using a linearized analysis which included the loss and gain processes. However, the result is perhaps not surprising since it is well known in quantum optics that this Hamiltonian will turn a coherent state into a squeezed state [11], which cannot be described by mixture of coherent states.

This result is interesting because the ignorance interpretation of the coherent state representation ("the laser is really in a coherent state, I just haven't been bothered to find out which one it is") is very commonly adopted in quantum optics (see Ref. [14] for a discussion). Although it is tenable in principle for most optical lasers where $\chi \ll 1$, it is not tenable for atom lasers where we expect $\chi \gg 1$ [13]. So although atom lasers may give a coherent output, the condensate cannot be meaningfully considered to be in a coherent state. The relation between output coherence, and the physical realizability of states of the condensate, will be explored elsewhere.

HMW gratefully acknowledges conversations with $\mathrm{T}$. Rudolph and R. Spekkens.

[1] B. d'Espagnat, Conceptual Foundations of Quantum Mechanics 2nd Ed. (Addison-Wesley, New York, 1989).

[2] P. Kok and S.L. Braunstein, Phys. Rev. A 61, 042304 (2000).

[3] C.W. Gardiner and P. Zoller, Quantum Noise (Springer, Berlin, 2000).

[4] The time scale for the measurement must be much longer than the correlation time of the environment, but much smaller than any relevant system dynamical time scale.

[5] L.P. Hughston, R. Josza, and W.K. Wootters, Phys. Lett. A 183, 14 (1993).

[6] C.W. Gardiner, Handbook of Stochastic Methods (Springer, Berlin, 1985).

[7] H.M. Wiseman and J.A. Vaccaro, Phys. Lett. A 250, 241 (1998). 
[8] A. Einstein, Phys. Zeits. 18, 121 (1917).

[9] L. Diósi and C. Kiefer, Phys. Rev. Lett. 85, 3552 (2000).

[10] H.M. Wiseman, Phys. Rev. A 56, 2068 (1997).

[11] D.F. Walls and G.J. Milburn, Quantum Optics (Springer, Berlin, 1994).
[12] W.H. Louisell, Quantum Statistical Properties of Radiation (John Wiley \& Sons, New York, 1973).

[13] H.M. Wiseman and J.A. Vaccaro, unpublished (but see quant-ph/9906125)

[14] K. Mølmer, Phys. Rev. A 55, 3195 (1996). 